\title{
Compactification of Classical Groups
}

\author{
HONGYU HE
}

\section{Introduction.}

\subsection{Historical Notes.}

Compactifications of symmetric spaces and their arithmetic quotients have been studied by many people from different perspectives. Its history went back to E. Cartan's original paper ([2]) on Hermitian symmetric spaces. Cartan proved that a Hermitian symmetric space of noncompact type can be realized as a bounded domain in a complex vector space. The compactification of Hermitian symmetric space was subsequently studied by Harish-Chandra and Siegel (see [16]). Thereafter various compactifications of noncompact symmetric spaces in general were studied by Satake (see [14]), Furstenberg (see [3]), Martin (see [4]) and others. These compactifications are more or less of the same nature as proved by C. Moore (see [11]) and by Guivarc'h-Ji-Taylor (see [4]). In the meanwhile, compactification of the arithmetic quotients of symmetric spaces was explored by Satake (see [15]), Baily-Borel (see [1]), and others. It plays a significant role in the theory of automorphic forms. One of the main problems involved is the analytic properties of the boundary under the compactification. In all these compactifications that have been studied so far, the underlying compact space always has boundary. For a more detailed account of these compactifications, see [16] and [4].

In this paper, motivated by the author's work on the compactification of symplectic group (see [9]), we compactify all the classical groups. One nice feature of our compactification is that the underlying compact space is a symmetric space of compact type. Furthermore, for real groups, real analytic structure is preserved; for complex groups, complex analytic structure is preserved (see Theorem 2.4 and Theorem 3.3). In addition, all the $G(p, q)$ groups for $p+q=n$ can be compactified simultaneously (see Theorem 2.3). I should remark that this compactification is essentially different from the construction in [13]. The main idea in this paper is due to D. Vogan ([17]). 
Throughout this paper, we will mainly follow the standard notations from Helgason's book [5] and Knapp's book [12].

\subsection{Compactification: Ideas.}

Let $X$ be a noncompact analytic manifold, $Y$ be a compact analytic manifold. We say that $(i, Y)$ is an analytic compactification of $X$ if $i$ is an analytic embedding from $X$ to $Y$ and $i(X)$ is open and dense in $Y$. We say that $(i, X)$ is a complex analytic compactification if in addition $i$ is complex analytic. In [9], I construct an analytic compactification $(\mathcal{H}, U(2 n) / O(2 n))$ of $S p_{2 n}(\mathbb{R})$ based on the integration kernel of the oscillator representation. The homogeneous space $U(2 m) / O(2 n)$ is taken to be the $2 n \times 2 n$ symmetric unitary matrices. In [9], the formula for $\mathcal{H}$ was laid out via $K A K$ decomposition of $S p_{2 n}(\mathbb{R})$. In [10], $\mathcal{H}$ was explicitly computed as fractions:

Theorem 0.1. Let $g=\left(\begin{array}{cc}A & B \\ C & D\end{array}\right) \in S p_{2 n}(\mathbb{R})$. Then $\mathcal{H}(g)=\left(\begin{array}{cc}\left(\frac{A^{t}+D^{t}}{2}-i \frac{C^{t}-B^{t}}{2}\right)^{-1}\left(\frac{A^{t}-D^{t}}{2}+i \frac{B^{t}+C^{t}}{2}\right) & -i\left(\frac{A^{t}+D^{t}}{2}-i \frac{C^{t}-B^{t}}{2}\right)^{-1} \\ -i\left(\frac{A+D}{2}-i \frac{C-B}{2}\right)^{-1} & \left(\frac{A+D}{2}-i \frac{C-B}{2}\right)^{-1}\left(\frac{A-D}{2}-i \frac{B+C}{2}\right)\end{array}\right)$.

In [10], I also showed that the left and right group action of $S p_{2 n}(\mathbb{R})$ can be extended to a left and right action on $U(2 n) / O(2 n)$.

Theorem 0.2. Let $G=S p_{2 n}(\mathbb{R})$. Let $U(\mathfrak{g})$ be the universal enveloping algebra of $\mathfrak{g}$. Let $L(U(\mathfrak{g}))$ be the space of right invariant differential operators on $G$, and $R(U(\mathfrak{g}))$ be the space of left invariant differential operators on $G$. Let $D$ be an differential operator in $L(U(\mathfrak{g})) \otimes R(U(\mathfrak{g}))$. Then $D$ can be extended to an algebraic differential operator on $\bar{G}$.

Now, one remaining problem is to classify all the bi- $S p_{2 n}(\mathbb{R})$ orbits on $U(2 n) / O(2 n)$. This problem seems unapproachable from our construction.

Another interesting problem is to compactify all the other classical groups. The analytic approach of compactification used in [9], [10] can be extended to other classical groups by using dual pairs (see [8]). Roughly speaking, a dual pair is a pair of classical groups embedded in a symplectic group as commuting subgroups (see [18], [19]). Therefore, a compactification of symplectic group will automatically yield a compactification of the dual pair. Even though the analytic approach would give us the explicit formula, the computation is expected to be intense. 
The geometry behind our compactification $(\mathcal{H}, U(2 n) / O(2 n))$ was discovered by Vogan ([17]). In fact, Vogan constructed a "new" compactification $(i, U(2 n) / O(2 n))$ by realizing $U(2 n) / O(2 n)$ as the Lagrangian Grassmannian of $\mathbb{R}^{4 n}$. It is believed that this "new" construction is exactly $(\mathcal{H}, U(2 n) / O(2 n))$ I constructed earlier. In this paper, I will apply Vogan's idea to compactify all the classical groups and identify $(i, U(2 n) / O(2 n))$ with $(\mathcal{H}, U(2 n) / O(2 n))$ for $S p_{2 n}(\mathbb{R})$ in the Appendix. Vogan's idea can be stated as follows $([17])$.

Let $H$ be a classical semisimple group. Let $\sigma$ be a group involution. Let $X=H / P$ be a generalized flag variety. Let $L$ be the set of fixed points of $\sigma$ ( $L$ may or may not be connected). Then it is well-known that $L$ acts on $X$ with finite number of orbits. In particular, when there is a unique open $L$-orbit, we call it the generic orbit. If we choose $(H, X, \sigma)$ such that the generic $L$-orbit exists and can be identified with our intended group $G$, then this construction will automatically produce an analytic compactification of $G$, namely $(i, X)$. The embedding $i$ is simply the identification of $G$ with the generic orbit. One nice feature that comes freely with this construction is the geometric interpretation. For example, the classification of $L$-orbits depends more or less on the geometry of the flags in $X$.

In this paper, we will give unified constructions of $(i, X)$ for classical groups of type I and II. The group $H$ will be a bigger classical group of the same type as $G$. $L$ will be $G \times G$ and $P$ will be a maximal parabolic subgroup of $H$. One necessary condition for $(i, X)$ to be an analytic compactification of $G$ is that the $L$ action on the generic orbit in $X$ must be identical to the left and right group action of $G$. This is consistent with our result in [10], that is, in the symplectic case, the left and right $G$ actions can be automatically extended to $X$. In this paper, we will further classify all the $G \times G$-orbits in $X$.

\subsection{Content and Results.}

Our paper is organized as follows. In Chapter 1, I construct the compactification $(i, X)$ for symplectic groups. Here $H=S p_{4 n}(\mathbb{R}), L=$ $S p_{2 n}(\mathbb{R}) \times S p_{2 n}(\mathbb{R})$ and $X$ is the Lagrangian Grassmannian of $\mathbb{R}^{4 n}$. I define the index of a Lagrangian subspace $X$ as an $L$-invariant and classify all the $L$-orbits based on this index. Most of the results in this chapter is due to Vogan. In Chapter 2, I generalize the construction in Chapter 1 to all the classical groups of type I (see Definition 0.1). The following in the main result. 
Theorem 0.3. Let $G$ be a classical group of type I. Let $r$ be the real rank of $G$. Then there exists a natural analytic compactification $(i, X)$ with

1. $X=O(n)$ for $G=O(p, n-p)$;

2. $X=U(p+q)$ for $G=U(p, q)$;

3. $X=U(2 n) / S p(n)$ for $G=O^{*}(2 n)$;

4. $X=S p(p+q)$ for $G=S p(p, q)$;

5. $X=O(2 n) / U(n)$ for $G=O(n, \mathbb{C})$;

6. $X=S p(2 n) / U(2 n)$ for $G=S p_{2 n}(\mathbb{C})$;

7. $X=U(2 n) / O(2 n)$ for $G=S p_{2 n}(\mathbb{R})$.

Furthermore, the number of $G \times G$-orbits on $X$ is equal to $r+1$.

In Chapter 3, we treat classical groups of type II, namely the general linear groups. The following is the main result.

Theorem 0.4. Let $G$ be a general linear group over $\mathbb{R}, \mathbb{C}$ or $\mathbb{H}$. Then there exists a natural analytic compactification $(i, X)$ with

1. $X=O(2 n) / O(n) \times O(n)$ for $G=G L(n, \mathbb{R})$;

2. $X=U(2 n) / U(n) \times U(n)$ for $G=G L(n, \mathbb{C})$;

3. $X=S p(2 n) / S p(n) \times S p(n)$ for $G=G L(n, \mathbb{H})$.

Furthermore, $G \times G$ acts on $X$ with $\frac{(n+1)(n+2)}{2}$ orbits.

For $G$ a complex classical group, $X$ possesses a natural complex structure. The complex structures are in fact preserved under our compactification.

Theorem 0.5. For $G=S p_{2 n}(\mathbb{C}), G L(n, \mathbb{C}), O(n, \mathbb{C})$, the compactification $(i, X)$ preserves the complex structure.

In Chapter 4, we make some remarks about some remaining questions that need to be addressed. In the Appendix, we show that for symplectic group, $(i, X)$ can be identified with $(\mathcal{H}, X)$ in [9]. The idea is as follows. We fix a complex structure and a complex inner product on $\mathbb{R}^{4 n}$ compatible with the symplectic structure. We observe that a real orthonormal basis in any Lagrangian subspace produces a complex orthonormal basis in $\mathbb{R}^{4 n}$. 
Based on this observation, we further identify Lagrangian subspaces of $\mathbb{R}^{4 n}$ with $2 n \times 2 n$ symmetric unitary matrices. We examine $(i, X)$ for $S p_{2 n}(\mathbb{R})$ under $K A K$ decomposition and prove that $i$ satisfies all the conditions that uniquely determine $\mathcal{H}$. Thus the compactification $\mathcal{H}$ in Theorem 0.1 and $i$ are identical.

\section{Compactification of Symplectic Group: Vogan's Construction.}

\subsection{Preliminary.}

A linear subspace of a symplectic space is said to be isotropic if the restriction of the symplectic form vanishes in such a subspace. Let $\mathcal{I}(2 n, m)$ be the set of $m$-dimensional isotropic subspaces of the standard $2 n$-dimensional symplectic space. If an isotropic subspace $V$ is half of the total dimension of the symplectic space, we say $V$ is a Lagrangian subspace. We denote the set of Lagrangian subspaces of the standard $2 n$-dimensional symplectic space by $\mathcal{L}(2 n)$. Then $\mathcal{I}(2 n, n) \cong \mathcal{L}(2 n)$. If the symplectic space $V$ is equipped with only one symplectic form $\omega$, we use $\mathcal{I}(V, m)$ to denote the set of $m$ dimensional isotropic subspaces of $(V, \omega)$, and $\mathcal{L}(V)$ to denote the set of Lagrangian subspaces of $(V, \omega)$.

Let $(V, \omega)$ be a symplectic space. Let us fix an isotropic subspace $V_{0}$. Let $V_{0}^{\perp}$ be the space of vectors which are perpendicular to $V_{0}$ under the bilinear form $\omega$. The sufficient and necessary condition for $V_{0}$ to be isotropic is

$$
V_{0}^{\perp} \supseteq V_{0}
$$

for $V_{0}$ to be Lagrangian is

$$
V_{0}^{\perp}=V_{0} .
$$

One consequence of $V_{0}$ being isotropic is that it uniquely determines a symplectic flag:

$$
\{0\} \subseteq V_{0} \subseteq V_{0}^{\perp} \subseteq V .
$$

Now we define a new symplectic form $\mu$ on $V_{0}^{\perp} / V_{0}$ as follows

$$
\mu\left(u+V_{0}, v+V_{0}\right)=\omega(u, v) .
$$

It is trivial to check that $\mu$ is a well-defined bilinear form and skewsymmetric. To see that $\mu$ is non-degenerate, suppose

$$
\mu\left(u+V_{0}, V_{0}^{\perp}\right)=\omega\left(u, V_{0}^{\perp}\right)=0 .
$$


Then $u+V_{0} \in\left(V_{0}^{\perp}\right)^{\perp}$. We have

$$
u+V_{0}=V_{0} \in V_{0}^{\perp} / V_{0} .
$$

Thus $u \equiv 0\left(\bmod V_{0}\right)$. This shows that $\mu$ is nondegenerate. Therefore $\mu$ is a symplectic form on $V_{0}^{\perp} / V_{0}$. We call $\mu$ the quotient (reduced) symplectic form of $\omega$ on $V_{0}^{\perp} / V_{0}$, usually denoted by $\omega_{V_{0}^{\perp} / V_{0}}$.

Let $S p_{2 n}(\mathbb{R})$ be the standard symplectic group, $\omega$ the standard symplectic form. Let $\left\{\chi_{1}, \chi_{2}, \ldots, \chi_{n}, e_{1}, e_{2}, \ldots, e_{n}\right\}$ be a basis of $\mathbb{R}^{2 n}$ such that

$$
\begin{gathered}
\omega\left(e_{i}, e_{j}\right)=\omega\left(\chi_{i}, \chi_{j}\right)=0, \\
\omega\left(\chi_{i}, e_{j}\right)=\delta_{i}^{j} .
\end{gathered}
$$

Let $X_{m}$ be the linear span of $\left\{\chi_{1}, \ldots, \chi_{m}\right\}$. Let $P_{m}$ be the standard parabolic subgroup stabilizing $X_{m}$. If we regard $\mathcal{I}(2 n, m)$ as a flag variety, we have

$$
\mathcal{I}(2 n, m) \cong S p_{2 n}(\mathbb{R}) / P_{m} .
$$

Let $N_{m}$ be the nilradical of $P_{m}$. Let $L_{m}$ be the Levi subgroup of $P_{m}$ which also stabilizes the linear span of $\left\{e_{1}, e_{2}, \ldots, e_{m}\right\}$. Then we have

$$
L_{m} \cong S p_{2(n-m)}(\mathbb{R}) \times G L_{m}(\mathbb{R})
$$

Notice that $P_{m}$ acts on $X_{m}^{\perp} / X_{m}$ preserving $\omega_{X_{\frac{m}{m}}} / X_{m}$. In fact, we have for $x, y \in X_{m}^{\perp}$ and $p \in P_{m}$

$$
\omega_{X_{m}^{\perp} / X_{m}}([x],[y])=\omega(x, y)=\omega(p x, p y)=\omega_{X_{\frac{1}{m}}^{\perp} X_{m}}([p x],[p y]) .
$$

Now it is easy to see that $P_{m}$ acts on $\left(X_{m}^{\perp} / X_{m}, \omega_{X_{m}^{\perp} / X_{m}}\right)$ via the $S p_{2(n-m)}(\mathbb{R})$ factor in $L_{m}$. We summarize these facts in the following lemma.

Lemma 1.1. We have

$$
\mathcal{I}(2 n, m) \cong S p_{2 n}(\mathbb{R}) / P_{m}
$$

Furthermore $P_{m}$ acts on $X_{m}^{\perp} / X_{m}$ via $P_{m} /\left(G L_{m}(\mathbb{R}) \times N_{m}\right)$. 


\subsection{Vogan's construction.}

Let $(U, \omega)$ and $(W, \omega)$ be two identical copies of the standard symplectic space of dimension $2 n$. Let $U \oplus W$ be the direct sum endowed with the following symplectic form

$$
\Omega\left(\left(u_{1}, w_{1}\right),\left(u_{2}, w_{2}\right)\right)=\omega\left(u_{1}, u_{2}\right)-\omega\left(w_{1}, w_{2}\right) \quad\left(u_{1}, u_{2} \in U ; w_{1}, w_{2} \in W\right) .
$$

Let $G_{1}$ be the symplectic group $S p(U, \omega)$ and $G_{2}$ be the symplectic group $S p(W, \omega)$. Then $G_{1} \times G_{2}$ acts on $U \oplus W$ diagonally. Furthermore $\Omega$ is fixed under the action of $G_{1} \times G_{2}$. We regard $G_{1} \times G_{2}$ as a subgroup of $S p(U \oplus W, \Omega)$.

Now let $\mathcal{L}(U \oplus W)$ be the Grassmannian of Lagrangian subspaces of $(U \oplus$ $W, \Omega)$. Then $\mathcal{L}(U \oplus W)$ can be identified with $S p(U \oplus W, \Omega) / G L(2 n, \mathbb{R}) N_{2 n}$ where $N_{2 n}$ consists of

$$
\left\{\left(\begin{array}{ll}
I_{2 n} & X \\
0_{2 n} & I_{2 n}
\end{array}\right) \quad X^{t}=X\right\}
$$

Firstly, since the maximal compact subgroup of $S p(U \oplus W, \Omega)$ is $U(2 n)$ and the maximal compact subgroup of $G L(2 n, \mathbb{R})$ is $O(2 n), \mathcal{L}(U \oplus W)$ can be identified with $U(2 n) / O(2 n)$. Secondly, Since $\mathcal{L}(U \oplus W)$ possesses a $S p(U \oplus W, \Omega)$-action, it possesses a $G_{1} \times G_{2}$ action.

Lemma 1.2. The compact symmetric space $U(2 n) / O(2 n)$ has a bi-Sp $2 n(\mathbb{R})$ action. These two $S p_{2 n}(\mathbb{R})$-actions commute.

For $V \in \mathcal{L}(U \oplus W)$, the $G_{1}$ action is given by

$$
g_{1} V=\left\{\left(g_{1} u, v\right) \mid(u, v) \in V\right\} .
$$

The $G_{2}$ action is given by

$$
g_{2} V=\left\{\left(u, g_{2} v\right) \mid(u, v) \in V\right\} .
$$

Apparently, $g_{1}$ commutes with $g_{2}$.

Now for each $g \in S p_{2 n}(\mathbb{R})$, we regard it as a linear function from $U$ to $W$. We consider the graph of $g$, denoted by $i(g)$. The graph $i(g)$ is a $2 n$-dimensional subspace of $U \oplus W$. Since

$$
\Omega((x, g x),(y, g y))=\omega(x, y)-\omega(g x, g y)=0 \quad(x \in U, g \in S p(U, W))
$$


$i(g)$ is a Lagrangian subspace of $(U \oplus W, \Omega)$. Therefore $i\left(S p_{2 n}(\mathbb{R})\right)$ is in $\mathcal{L}(U \oplus W)$. We may identify $g$ with $i(g)$. It is easy to see that $i\left(S p_{2 n}(\mathbb{R})\right)$ is a single $G_{1} \times G_{2}$-orbit.

In the next section, we will show that $i\left(S p_{2 n}(\mathbb{R})\right)$ is the generic $G_{1} \times G_{2^{-}}$ orbit in $\mathcal{L}(U \oplus W)$. We will also classify the orbital structure of $G_{1} \times G_{2}$ on $\mathcal{L}(U \oplus W)$ and study each orbit. Very briefly, for a nongeneric orbit, we want to single out the degenerate part of each Lagrangian subspace and construct a fibration such that the fiber is "generic" with respect to certain reduced symplectic form.

\subsection{Index.}

Let $V$ be a Lagrangian subspace of $(U \oplus W, \Omega)$. We define

$$
V_{1}=V \cap U \quad V_{2}=V \cap W .
$$

Then $V_{1}$ and $V_{2}$ are isotropic subspaces of $(U \oplus W, \Omega)$. Since the restrictions of $\Omega$ on $U$ and $W$ are just $\omega$ and $-\omega, V_{1}$ and $V_{2}$ are isotropic subspaces of $(U, \omega)$ and $(W, \omega)$ respectively. Let $V_{1}^{\perp}$ be the subspace of $U$ perpendicular to $V_{1}$ with respect $\omega$. Let $V_{2}^{\perp}$ be the subspace of $W$ perpendicular to $V_{2}$ with respect to $\omega$. Then we have

$$
V_{i} \subset V_{i}^{\perp}
$$

We denote the projection $U \oplus W \rightarrow U$ by $\pi_{1}$, and $U \oplus W \rightarrow W$ by $\pi_{2}$. The map $\pi_{1} \oplus \pi_{2}$ is just the identity map. We have the following lemma

Lemma 1.3. Suppose $V$ is a Lagrangian subspace of $(U \oplus W, \Omega)$ and $V_{1}=U \cap V, V_{2}=W \cap V$. Then we have

$$
\begin{gathered}
\operatorname{ker}\left(\left.\pi_{1}\right|_{V}\right)=V_{2} \quad \operatorname{ker}\left(\left.\pi_{2}\right|_{V}\right)=V_{1} \quad(\text { trivial }), \\
\pi_{1}(V)=V_{1}^{\perp} \quad \pi_{2}(V)=V_{2}^{\perp} \\
\operatorname{dim} V_{1}=\operatorname{dim} V_{2} .
\end{gathered}
$$

Proof. Since $V$ is Lagrangian, for every $v \in V$, we must have

$$
\Omega\left(\left(\pi_{1}(v), \pi_{2}(v)\right), V \cap U\right)=0 .
$$

This implies that $\omega\left(\pi_{1}(v), V_{1}\right)=0$. Therefore we have

$$
\pi_{1}(v) \in V_{1}^{\perp} .
$$


It follows that

$$
\pi_{1}(V) \subseteq V_{1}^{\perp}
$$

Similarly, we have

$$
\pi_{2}(V) \subseteq V_{2}^{\perp} .
$$

By taking the dimensions, we obtain

$$
\operatorname{dim}\left(\pi_{i}(V)\right) \leq 2 n-\operatorname{dim}\left(V_{i}\right) \quad(i=1,2) .
$$

On the other hand, since $V_{1}$ is the kernel of $\left.\pi_{2}\right|_{V}$, and $V_{2}$ is the kernel of $\left.\pi_{1}\right|_{V}$, we have

$$
\operatorname{dim}\left(\pi_{1}(V)\right)=\operatorname{dim} V-\operatorname{dim} V_{2} \quad \operatorname{dim}\left(\pi_{2}(V)\right)=\operatorname{dim} V-\operatorname{dim} V_{1} .
$$

Combined with Equation 3, we obtain

$$
\begin{aligned}
& 2 n-\operatorname{dim}\left(V_{2}\right)=\operatorname{dim}\left(\pi_{1}(V)\right) \leq 2 n-\operatorname{dim}\left(V_{1}\right), \\
& 2 n-\operatorname{dim}\left(V_{1}\right)=\operatorname{dim}\left(\pi_{2}(V)\right) \leq 2 n-\operatorname{dim}\left(V_{2}\right) .
\end{aligned}
$$

These inequalities force

$$
\begin{gathered}
\operatorname{dim}\left(V_{1}\right)=\operatorname{dim}\left(V_{2}\right), \\
\operatorname{dim}\left(\pi_{1}(V)\right)=\operatorname{dim}\left(V_{1}^{\perp}\right) \quad \operatorname{dim}\left(\pi_{2}(V)\right)=\operatorname{dim}\left(V_{2}^{\perp}\right) .
\end{gathered}
$$

Combined with Equations 1 and 2, we obtain

$$
\begin{gathered}
\pi_{1}(V)=V_{1}^{\perp} \quad \pi_{2}(V)=V_{2}^{\perp}, \\
\operatorname{dim}\left(V_{1}\right)=\operatorname{dim}\left(V_{2}\right)=2 n-\operatorname{dim}\left(\pi_{1}(V)\right)=2 n-\operatorname{dim}\left(\pi_{2}(V)\right) .
\end{gathered}
$$

Definition 1.1. Suppose $V$ is a Lagrangian subspace of $(U \oplus W, \Omega)$. We call the dimension of $V_{1}$ or $V_{2}$, the index of $V$. This defines a map

$$
\text { ind }: \mathcal{L}(U \oplus W) \rightarrow \mathbb{N} \text {. }
$$

Lemma 1.4. Let $V \in \mathcal{L}(U \oplus W)$. The following are equivalent:

- $\operatorname{ind}(V)=i$; 
- $\operatorname{dim}(V \cap U)=i$;

- $\operatorname{dim}(V \cap W)=i$;

- $\operatorname{dim}\left(\pi_{1}(V)\right)=2 n-i$;

- $\operatorname{dim}\left(\pi_{2}(V)=2 n-i\right.$.

Let $\mathcal{L}(U \oplus W)_{i}$ be the set of Lagrangian subspaces with index $i$. We observe that for any $g_{1} \in G_{1}, g_{2} \in G_{2}$,

$$
\begin{gathered}
i n d\left(g_{1} V\right)=\operatorname{dim}\left(g_{1} V \cap U\right)=\operatorname{dim}\left(V \cap g_{1}^{-1} U\right)=\operatorname{dim}(V \cap U)=\operatorname{ind}(V), \\
\operatorname{ind}\left(g_{2} V\right)=\operatorname{dim}\left(g_{2} V \cap W\right)=\operatorname{dim}\left(V \cap g_{2}^{-1} W\right)=\operatorname{dim}(V \cap W)=i n d(V) .
\end{gathered}
$$

We obtain

Lemma 1.5. The index of $V$ is preserved by the action of $G_{1} \times G_{2}$.

\subsection{Generic Orbit.}

The definition of index will help us nail down the image of

$$
i: S p_{2 n}(\mathbb{R}) \rightarrow \mathcal{L}(U \oplus W)
$$

we defined earlier. The following theorem says that $i$ produces a one to one correspondence between $\mathcal{L}(U \oplus W)_{0}$ and $S p_{2 n}(\mathbb{R})$.

Theorem 1.1 (Vogan). The set $\mathcal{L}(U \oplus W)_{0}$ can be identified with $S p_{2 n}(\mathbb{R})$ through $i$. This identification $i$ preserves the $S p_{2 n}(\mathbb{R}) \times S p_{2 n}(\mathbb{R})$ action. Therefore for $V \in \mathcal{L}(U \oplus W)_{0}$, the isotropic subgroup $\left(G_{1} \times G_{2}\right)_{V}$ is isomorphic to the subgroup

$$
\left\{(g, g) \mid g \in S p_{2 n}(\mathbb{R})\right\} .
$$

Proof. Let $V \in \mathcal{L}(U \oplus W)_{0}$. Then we have

$$
V \cap U=\{0\} \quad V \cap W=\{0\} .
$$

Therefore $\left.\pi_{1}\right|_{V}$ and $\left.\pi_{2}\right|_{V}$ are all injections. But $\operatorname{dim}(V)=\operatorname{dim}(U)=$ $\operatorname{dim}(W)$. Thus $\left.\pi_{1}\right|_{V}$ and $\left.\pi_{2}\right|_{V}$ are isomorphisms. This implies that $V$ is the graph of a one-to-one correspondence

$$
\phi_{V}: U \rightarrow W .
$$


In fact, $\phi_{V}$ can be written explicitly as

$$
\pi_{2} \circ\left(\left.\left(\pi_{1}\right)\right|_{V}\right)^{-1} .
$$

Now we know that

$$
V=\left\{\left(x, \phi_{V}(x)\right) \mid x \in U\right\} .
$$

Since $V$ is Lagrangian, for any $x, y \in U$, we must have

$$
0=\Omega\left(\left(x, \phi_{V}(x)\right),\left(y, \phi_{V}(y)\right)\right)=\omega(x, y)-\omega\left(\phi_{V}(x), \phi_{V}(y)\right) .
$$

This means that $\phi_{V} \in S p(U, W) \cong S p_{2 n}(\mathbb{R})$. Conversely, for each $g \in$ $S p_{2 n}(\mathbb{R})$, we can define $V$ to be $i(g)$, the graph of $g$. Then $V$ is a Lagrangian subspace of $(U \oplus W, \Omega)$ and its index is 0 . Furthermore, it is easy to check that

$$
\phi_{i(g)}=g \quad i\left(\phi_{V}\right)=V .
$$

Therefore $i$ defines one-to-one correspondence between $S p_{2 n}(\mathbb{R})$ and $\mathcal{L}(U \oplus W)_{0}$. Now let $g_{1} \in G_{1}$ and $g_{2} \in G_{2}$. Then

$$
i\left(g_{1} g g_{2}\right)=\left\{\left(x, g_{1} g g_{2} x\right) \mid x \in U\right\}=\left\{\left(g_{2}^{-1} x, g_{1} g x\right) \mid x \in U\right\}=g_{1} g_{2}^{-1} i(g) .
$$

Hence $i$ preserves the $S p_{2 n}(\mathbb{R}) \times S p_{2 n}(\mathbb{R})$ action. Let $e$ be the identity element in $S p_{2 n}(\mathbb{R})$. Apparently, the subgroup stabilizing $i(e)$ is given by

$$
\left(G_{1} \times G_{2}\right)_{X} \cong\left\{(g, g) \mid g \in S p_{2 n}(\mathbb{R})\right\} .
$$

\subsection{Nongeneric Orbits.}

Let us consider $\mathcal{L}(U \oplus W)_{i}$ for $i>0$. We equip $\left(V_{1}^{\perp} \oplus V_{2}^{\perp}\right) /\left(V_{1} \oplus V_{2}\right)$ with the quotient symplectic form of $\Omega$. It is easy to see that

$$
\Omega_{\left(V_{1}^{\perp} \oplus V_{2}^{\perp}\right) /\left(V_{1} \oplus V_{2}\right)}=\omega_{V_{1}^{\perp} / V_{1}}-\omega_{V_{2}^{\perp} / V_{2}} .
$$

Theorem 1.2. There exists a fibration:

$$
\mathcal{L}\left(V_{1}^{\perp} / V_{1} \oplus V_{2}^{\perp} / V_{2}\right)_{0} \rightarrow \mathcal{L}(U \oplus W)_{i} \rightarrow \mathcal{I}(U, i) \times \mathcal{I}(W, i) .
$$

Proof. Let $V \in \mathcal{L}(U \oplus W)_{i}$. We define the projection of the fibration

$$
\pi(V)=\left(V_{1}, V_{2}\right)=(V \cap U, V \cap W) .
$$


This yields a surjection

$$
\pi: \mathcal{L}(U \oplus W)_{i} \rightarrow \mathcal{I}(U, i) \times \mathcal{I}(W, i) .
$$

Now fixing $V_{1} \in \mathcal{I}(U, i)$ and $V_{2} \in \mathcal{I}(W, i)$, we would like to study the fiber $\pi^{-1}\left(V_{1}, V_{2}\right)$. We wish to identify this fiber with $\mathcal{L}\left(V_{1}^{\perp} / V_{1} \oplus V_{2}^{\perp} / V_{2}\right)_{0}$. Recall from Lemma 1.3 that

$$
\pi_{1}(V)=V_{1}^{\perp} \quad \pi_{2}(V)=V_{2}^{\perp} .
$$

We obtain

$$
V \subseteq V_{1}^{\perp} \oplus V_{2}^{\perp} .
$$

Since $V_{1}=\operatorname{ker}\left(\left.\pi_{2}\right|_{V}\right)$ and $V_{2}=\operatorname{ker}\left(\left.\pi_{1}\right|_{V}\right)$, We obtain two isomorphisms:

$$
\begin{aligned}
& \pi_{1}: V / V_{2} \rightarrow V_{1}^{\perp}, \\
& \pi_{2}: V / V_{1} \rightarrow V_{2}^{\perp} .
\end{aligned}
$$

These two isomporhisms induces other two isomorphisms:

$$
\begin{aligned}
& \tilde{\pi_{1}}: V / V_{1} \oplus V_{2} \rightarrow V_{1}^{\perp} / V_{1}, \\
& \tilde{\pi_{2}}: V / V_{1} \oplus V_{2} \rightarrow V_{2}^{\perp} / V_{2} .
\end{aligned}
$$

Apparently, the direct sum

$$
\tilde{\pi_{1}} \oplus \tilde{\pi_{2}}: V / V_{1} \oplus V_{2} \rightarrow\left(V_{1}^{\perp} / V_{1}\right) \oplus\left(V_{2}^{\perp} / V_{2}\right)
$$

is also an isomorphism. We check the dimension

$$
\begin{aligned}
\operatorname{dim}\left(V / V_{1} \oplus V_{2}\right) & =\operatorname{dim}\left(V_{1}^{\perp}\right)-\operatorname{dim}\left(V_{2}\right) \\
& =\operatorname{dim}\left(V_{1}^{\perp}\right)-\operatorname{dim}\left(V_{1}\right)=\operatorname{dim}\left(V_{2}^{\perp}\right)-\operatorname{dim}\left(V_{2}\right) .
\end{aligned}
$$

We see that $V / V_{1} \oplus V_{2}$ is half of the dimension of $\left(V_{1}^{\perp} / V_{1}\right) \oplus\left(V_{2}^{\perp} / V_{2}\right)$. In fact, $V / V_{1} \oplus V_{2}$ is a Lagrangian subspace of $\left(V_{1}^{\perp} / V_{1}\right) \oplus\left(V_{2}^{\perp} / V_{2}\right)$. We prove this claim in two steps.

\section{Lemma 1.6.}

Let $\mu$ be the quotient symplectic form of $\Omega$ on $\left(V_{1}^{\perp} \oplus V_{2}^{\perp}\right) /\left(V_{1} \oplus V_{2}\right)$. Then $V /\left(V_{1} \oplus V_{2}\right)$ is an isotropic subspace of $\left(V_{1}^{\perp} / V_{1}\right) \oplus\left(V_{2}^{\perp} / V_{2}\right)$ with respect to $\mu$. 
Proof. For any $x, y \in V$, we know

$$
\left(\pi_{1} \oplus \pi_{2}\right)(x),\left(\pi_{1} \oplus \pi_{2}\right)(y) \in V_{1}^{\perp} \oplus V_{2}^{\perp} .
$$

Therefore, we have

$$
\mu\left(x+\left(V_{1} \oplus V_{2}\right), y+\left(V_{1} \oplus V_{2}\right)\right)=\omega(x, y)=0 .
$$

Thus $V /\left(V_{1} \oplus V_{2}\right)$ is an isotropic subspace of $\left(V_{1}^{\perp} / V_{1}\right) \oplus\left(V_{2}^{\perp} / V_{2}\right)$. This ends the proof of the lemma.

We observe that

$$
\left(V /\left(V_{1} \oplus V_{2}\right)\right) \cap\left(\left(V_{1}^{\perp} \oplus V_{2}\right) /\left(V_{1} \oplus V_{2}\right)\right)=\left(V_{1} \oplus V_{2}\right) /\left(V_{1} \oplus V_{2}\right)
$$

and

$$
\left(V /\left(V_{1} \oplus V_{2}\right)\right) \cap\left(\left(V_{1} \oplus V_{2}^{\perp}\right) /\left(V_{1} \oplus V_{2}\right)\right)=\left(V_{1} \oplus V_{2}\right) /\left(V_{1} \oplus V_{2}\right) .
$$

Combined with Equation 4, we obtain

Lemma 1.7. $V /\left(V_{1} \oplus V_{2}\right)$ is a Lagrangian subspace of $\left(V_{1}^{\perp} / V_{1}\right) \oplus\left(V_{2}^{\perp} / V_{2}\right)$. Furthermore,

$$
V /\left(V_{1} \oplus V_{2}\right) \in \mathcal{L}\left(\left(V_{1}^{\perp} / V_{1}\right) \oplus\left(V_{2}^{\perp} / V_{2}\right)\right)_{0} .
$$

Based on these two lemmas, we see that the fiber $\pi^{-1}\left(V_{1}, V_{2}\right)$ is contained in $\mathcal{L}\left(V_{1}^{\perp} / V_{1} \oplus V_{2}^{\perp} / V_{2}\right)_{0}$. Conversely, for any Lagrangian subspace

$$
X \in \mathcal{L}\left(\left(V_{1}^{\perp} / V_{1}\right) \oplus\left(V_{2}^{\perp} / V_{2}\right)\right)_{0}
$$

we define $V$ to be the preimage of $X$ under the projection

$$
V_{1}^{\perp} \oplus V_{2}^{\perp} \rightarrow\left(V_{1}^{\perp} / V_{1}\right) \oplus\left(V_{2}^{\perp} / V_{2}\right) .
$$

Then it is easy to verify that $V \in \mathcal{L}(U \oplus W)$ and $\operatorname{ind}(V)=i$. This ends the proof that the fiber of $\pi$ is exactly

$$
\mathcal{L}\left(V_{1}^{\perp} / V_{1} \oplus V_{2}^{\perp} / V_{2}\right)_{0} .
$$

Based on our established knowledge on the geometric structure of $\mathcal{L}(U \oplus W)_{i}$, we can analyze $\mathcal{L}(U \oplus W)_{i}$ in terms of the group action. Let

$$
\left\{e_{1}, e_{2}, \ldots, e_{2 n}\right\}
$$

be the standard basis for the symplectic space $\left(\mathbb{R}^{2 n}, \omega\right)$. Let $X_{i}$ be the linear span of $\left\{e_{1}, e_{2}, \ldots, e_{n}\right\}$. Apparently, $X_{i}$ is isotropic for $i \leq n$. 
Theorem 1.3. Each $\mathcal{L}(U \oplus W)_{i}$ is a single $G_{1} \times G_{2}$ orbit. Let $P_{i}$ be the parabolic subgroup stabilizing $X_{i}$ and $N_{i}$ be its nilradical. We identify the Levi subgroup $L_{i}$ with $S p_{2 n-2 i}(\mathbb{R}) \times G L_{i}(\mathbb{R})$. Let $Q$ be the subgroup of $P_{i} \times P_{i}$ consisting of

$$
\left\{\left(g g_{2} g_{3}, g h_{2} h_{3}\right) \mid g \in S p_{2 n-2 i}(\mathbb{R}), g_{2}, h_{2} \in G L_{i}(\mathbb{R}), g_{3}, h_{3} \in N_{i}\right\} .
$$

Then we have

$$
\mathcal{L}(U \oplus W)_{i} \cong S p_{2 n}(\mathbb{R}) \times S p_{2 n}(\mathbb{R}) / Q .
$$

Proof. Suppose $V \in \mathcal{L}(U \oplus W)_{i}$. Since $G_{1} \times G_{2}$ acts transitively on $\mathcal{I}(U, i) \times \mathcal{I}(W, i)$, without loss of generalities, we assume $X_{i}=V \cap U=$ $V \cap W$. The isotropic subgroup stabilizing $\left(X_{i}, X_{i}\right)$ is simply $P_{i} \times P_{i}$. Now $P_{i} \times P_{i}$ acts on $\mathcal{L}\left(\left(X_{i}^{\perp} / X_{i}\right) \oplus\left(X_{i}^{\perp} / X_{i}\right)\right)_{0}$. The left $P_{i}$ acts on $U / X_{i}$ via $P_{i} / G L_{i}(\mathbb{R}) N_{i} \cong S p_{2 n-2 i}(\mathbb{R})$ and the right $P_{i}$ acts on $W / X_{i}$ in the same way. From Lemma 1.1, $\mathcal{L}\left(\left(X_{i}^{\perp} / X_{i}\right) \oplus\left(X_{i}^{\perp} / X_{i}\right)\right)_{0}$ can be identified with $S p_{2 n-2 i}(\mathbb{R})$. Thus $P_{i}$ actions on $\mathcal{L}\left(\left(X_{i}^{\perp} / X_{i}\right) \oplus\left(X_{i}^{\perp} / X_{i}\right)\right)_{0}$ descend into left and right $S p_{2 n-2 i}(\mathbb{R})$ actions on $S p_{2 n-2 i}(\mathbb{R})$. It follows that both actions of $P_{i}$ are transitive.

From the fibration

$$
\mathcal{L}\left(V_{1}^{\perp} / V_{1} \oplus V_{1}^{\perp} / V_{1}\right)_{0} \rightarrow \mathcal{L}(U \oplus W)_{i} \rightarrow G_{1} / P_{i} \times G_{2} / P_{i}
$$

we see that $\mathcal{L}(U \oplus W)_{i}$ is a single $G_{1} \times G_{2}$ orbit. Furthermore, for $\tilde{\pi_{1}} \oplus \tilde{\pi_{2}}(V) \in \mathcal{L}\left(\left(X_{i}^{\perp} / X_{i}\right) \oplus\left(X_{i}^{\perp} / X_{i}\right)\right)_{0}$, from Theorem 1.1, the isotropy group

$$
\left(P_{i} \times P_{i}\right)_{\tilde{\pi_{1} \oplus \tilde{\pi}}(V)}
$$

is isomorphic to $Q$. Therefore the isotropic group of $G_{1} \times G_{2}$ action on $V$ is isomorphic to

$$
\left(P_{i} \times P_{i}\right)_{\tilde{\pi_{1} \oplus \tilde{\pi}}(V)} \cong Q
$$

The dimension of $\mathcal{L}(U \oplus W)_{i}$ is equal to

$$
\begin{aligned}
& 2 \operatorname{dim}(\mathcal{I}(U, i))+\operatorname{dim}\left(\mathcal{L}(4 n-4 i)_{0}\right) \\
& =2\left(\operatorname{dim}\left(S p_{2 n}(\mathbb{R})\right)-\operatorname{dim}\left(P_{i}\right)\right)+\operatorname{dim}\left(S p_{2 n-2 i}(\mathbb{R})\right) \\
& =\operatorname{dim}\left(S p_{2 n}(\mathbb{R})\right)-\operatorname{dim}\left(L_{i}\right)+\operatorname{dim}\left(S p_{2 n-2 i}(\mathbb{R})\right) \\
& =\operatorname{dim}\left(S p_{2 n}(\mathbb{R})\right)-\operatorname{dim}\left(G L_{i}(\mathbb{R})\right) \\
& \left.<\operatorname{dim}\left(\mathcal{L}(U \oplus W)_{0}\right)\right) .
\end{aligned}
$$


Therefore, $\mathcal{L}(U \oplus W)_{0}$ is an open dense subset of $\mathcal{L}(U \oplus W)$. We obtain the following theorem

Theorem 1.4. The map $i: S p_{2 n}(\mathbb{R}) \rightarrow \mathcal{L}(U \oplus W)$ defined by

$$
i(g)=\{(x, g x) \mid x \in U\}
$$

is an analytic compactification of $S p_{2 n}(\mathbb{R})$.

Proof. Let $e$ be the identity in $S p_{2 n}(\mathbb{R})$. First of all, $S p_{2 n}(\mathbb{R})$ acts on $\mathcal{L}(U \oplus$ $W)$ from left analytically. Therefore, the orbit $i\left(S p_{2 n}(\mathbb{R})\right)$ is an analytic submanifold. Since $i$ is one-to-one, $i$ is an analytic embedding. Furthermore, $i\left(S p_{2 n}(\mathbb{R})\right)$ is open and dense in $\mathcal{L}(U \oplus W)$. Hence $(i, \mathcal{L}(U \oplus W))$ is an analytic compactification.

\section{Compactification of Classical Groups of Type I.}

The techniques used in the last chapter to compactify the symplectic group can be easily generalized to all the classical groups of type I without much modification. We will give the definition of type I classical groups first, and then we will state our results. Since the proof of these results is not much different from the case for symplectic group, we will be brief.

\subsection{Classical Group of Type I.}

Definition 2.1 (Classical Group of Type I). A type I classical group $G(V)$ consists of the following data (see [7], [18], [19]).

- A division algebra $D$ of a field $\mathbb{F}$ with involution $\sharp$, and $a^{\sharp} b^{\sharp}=(b a)^{\sharp}$;

- A (right) vector space $V$ over $D$, with a nondegenerate ( $D$-valued) sesquilinear form $(,)_{\epsilon}, \epsilon= \pm 1$, i.e.,

$$
\begin{gathered}
(u, v)=\epsilon(v, u)^{\sharp} \quad(u, v \in V), \\
(u \lambda, v)=(u, v) \lambda \quad(u, v \in V, \lambda \in D) ;
\end{gathered}
$$

- $\mathrm{G}$ is the isometry group of $($,$) , i.e.,$

$$
\begin{gathered}
g \cdot(u \lambda)=(g . u) \lambda \quad(\lambda \in D, u \in V, g \in G) \\
(g u, g v)=(u, v) \quad(u, v \in V) .
\end{gathered}
$$


If $(V,()$,$) is implicitly understood, we denote the classical group by G$. In general, we denote it by $G(V)$. We allow $\sharp$ to be trivial. We call the identity component of $G$ connected classical group of type I. For $\mathbb{F}=\mathbb{C}$, trivial, we obtain all the complex classical groups of type I, namely, $S p_{2 n}(\mathbb{C})$, and $O(n, \mathbb{C})$. For $\mathbb{F}=\mathbb{R}, D=\mathbb{H}, \sharp$ the usual involution, we obtain $S p(p, q)$ and $O^{*}(2 n)$ depending on the sesquilinear form. For $\mathbb{F}=\mathbb{R}, D=\mathbb{C}$ and $\sharp$ the usual conjugation, we obtain $U(p, q)$ depending on the signature of the Hermitian form. For $\mathbb{F}=\mathbb{R}, D=\mathbb{R}$ with trivial involution, we obtain $S p_{2 n}(\mathbb{R})$ and $O(p, q)$.

Let $V_{0}$ be a linear subspace of $V$. We write $V_{0}^{\perp}$ for the orthogonal complement of $V_{0}$ with respect to the sesquilinear form (,). From now on, all the linear spaces and dimensions and homomorphism will be over $D$ unless stated otherwise. In some situation, a linear subspace refers to a point in a Grassmannian manifold depending on the context.

For $V=D^{n}$ endowed with a sesquilinear form (,), we say that a subspace $V_{0}$ is isotropic if

$$
\left(V_{0}, V_{0}\right)=0 \text {. }
$$

Let $r$ be the real rank of $G(n)$. Let $\left\{e_{1}, e_{2}, \ldots, e_{r}\right\}$ be a basis of a maximal isotropic subspace of $V$. Let $X_{i}$ be the linear span of $\left\{e_{1}, e_{2}, \ldots, e_{i}\right\}$. Let $P_{i}$ be the parabolic subgroup of $G(V)$ stabilizing the subspace $X_{i}$. Let $\mathcal{I}(V, i)$ be the set of $i$-dimensional isotropic subspaces of $V$. Then

$$
\mathcal{I}(V, i) \cong G(n) / P_{i}
$$

According to our definition of type I classical group, $P_{i}$ also stabilizes $X_{i}^{\perp}$. In fact, we have

$$
0=\left(X_{i}^{\perp}, X_{i}\right)=\left(X_{i}^{\perp}, P_{i} X_{i}\right)=\left(P_{i} X_{i}^{\perp}, X_{i}\right) .
$$

Therefore $P_{i}$ acts on $X_{i}^{\perp} / X_{i}$.

Now we fix an $i$. We define a nondegenerate sesquilinear form $\mu$ on $X_{i}^{\perp} / X_{i}$

$$
\mu\left(x+X_{i}, y+X_{i}\right)=(x, y) \quad\left(x, y \in X_{i}^{\perp}\right) .
$$

We call $\mu$ the reduced sesquilinear form of $($,$) , denoted by (,)_{X_{i}^{\perp} / X_{i}}$. Now the $P_{i}$ action on $X_{i}^{\perp} / X_{i}$ descends to the group action of $G\left(X_{i}^{\perp} / X_{i},(,)_{X_{i}^{\perp} / X_{i}}\right)$. More precisely, let $N_{i}$ be the nilradical of $P_{i}$. Then $P_{i} / N_{i}$ can be identified with $G\left(X_{i}^{\perp} / X_{i},(,)_{X_{i}^{\perp} / X_{i}}\right) \times G L(i, D)$ and apparently $G L(i, D)$ acts trivially on $X_{i}^{\perp} / X_{i}$. Therefore $P_{i}$ acts on $X_{i}^{\perp} / X_{i}$ via $P_{i} / N_{i} \times G L(n, D)$. For more details on the structure of $P_{i}$. We refer the reader to Jian-Shu Li's paper [7]. 


\subsection{Main Theorem I.}

Let $U$ and $W$ be two identical copies of $D^{n}$ equipped with the same sesquilinear form (, ). Let $U \oplus W$ be the direct sum of $U$ and $W$ equipped with the following sesquilinear form

$$
\Omega\left(\left(u_{1}, w_{1}\right),\left(u_{2}, w_{2}\right)\right)=\left(u_{1}, u_{2}\right)-\left(w_{1}, w_{2}\right) \quad\left(u_{i} \in U, w_{i} \in W\right)
$$

Then $G(U) \times G(W)$ acts diagonally on $U \oplus W$. We say a subspace $V$ is a Lagrangian subspace of $U \oplus W$ if

$$
\Omega(V, V)=0 \quad \operatorname{dim}_{D} V=n .
$$

Let $\mathcal{L}(U \oplus W)$ be the set of Lagrangian subspaces of $U \oplus W$. Then $G(U) \times$ $G(W)$ acts on $\mathcal{L}(U \oplus W)$. Furthermore, we have

$$
\mathcal{L}(U \oplus W) \cong G(2 n) / P_{n}
$$

We define a map $i: G(n) \rightarrow L(U \oplus W)$ by

$$
i(g)=\left\{(v, g v) \mid v \in D^{n}\right\}
$$

Then $i(g) \in \mathcal{L}(U \oplus W)$.

Theorem 2.1 (Main Theorem-Type I). Let $G(n)$ be a classical group of type $I$. Let $r$ be the real rank of $G(n)$. Let $\mathcal{L}\left(D^{2 n}\right)$ be the set of Lagrangian subspaces of the following sesquilinear form

$$
\Omega\left(\left(u_{1}, w_{1}\right),\left(u_{2}, w_{2}\right)\right)=\left(u_{1}, u_{2}\right)-\left(w_{1}, w_{2}\right) \quad\left(u_{i}, w_{i} \in D^{n}\right)
$$

Then $G(n) \times G(n)$ acts on $\mathcal{L}\left(D^{2 n}\right)$ with $r+1$ orbits. There exists a unique open dense orbit $\mathcal{L}\left(D^{2 n}\right)_{0}$ (to be defined) which can be identified with $G(n)$ naturally.

Recall that $\mathcal{L}(U \oplus W)$ can be identified with $G(U \oplus W) / P_{n}$. Here $P_{n}$ is a parabolic subgroup stabilizing a Lagrangian subspace of $(U \oplus W, \Omega)$. We choose a $K A N$ decomposition of $G(U \oplus W)$ (warning, not of $G(n)$ ) such that the corresponding minimal parabolic group $M A N$ is contained in $P_{n}$. Let $M_{n} A_{n} N_{n}$ be the Langlands decomposition of $P_{n}$ (see $\mathrm{Ch}$. V in [12]). Then $\mathcal{L}(U \oplus W)$ can be identified with $K / M_{n} \cap K$. We observe that $M_{n}$ can always be identified with $G L(n, D)$ (see [7] and [8]). Therefore $M_{n} \cap K$ 
will be one of the compact classical groups, namely $O(n), U(n)$ or $S p(n)$. We obtain the following list:

(6)

$\begin{array}{ccccc}G(n) & G(U \oplus W) & K & G L(n, D) \cap K & \mathcal{L}(U \oplus W) \\ O(p, n-p) & O(n, n) & O(n) \times O(n) & O(n) & O(n) \times O(n) / O(n) \\ U(p, n-p) & U(n, n) & U(n) \times U(n) & U(n) & U(n) \times U(n) / U(n) \\ S p(p, n-p) & S p(n, n) & S p(n) \times S p(n) & S p(n) & S p(n) \times S p(n) / S p(n) \\ O^{*}(2 n) & O^{*}(4 n) & U(2 n) & S p(n) & U(2 n) / S p(n) \\ O(n, \mathbb{C}) & O(2 n, \mathbb{C}) & O(2 n) & U(n) & O(2 n) / U(n) \\ n \text { even, } S p_{n}(\mathbb{C}) & S p_{2 n}(\mathbb{C}) & S p(n) & U(n) & S p(n) / U(n) \\ n \text { even, } S p_{n}(\mathbb{R}) & S p_{2 n}(\mathbb{R}) & U(n) & O(n) & U(n) / O(n)\end{array}$

In each case $\mathcal{L}(U \oplus W)$ is a compact symmetric space (see [5]).

Theorem 2.2 (Compactification of Type I Classical Groups). For every classical group of type $I$, there exists an analytic compactification $(i, X)$ where $X$ is a compact symmetric space.

For the first three cases, in $\mathcal{L}(U \oplus W), O(n), U(n)$ and $S p(n)$ are embedded diagonally in their products. For any Lie group $G$, let $\Delta$ be the diagonal embedding of $G$ into $G \times G$. We can define an identification of $G \times G / \Delta(G)$ with $G$ as follows

$$
m:\left(g_{1}, g_{2}\right) \rightarrow g_{1} g_{2}^{-1} .
$$

Thus we obtain a canonical identification of $\mathcal{L}(U \oplus W)$ with $O(n), U(n)$ or $S p(n)$ in each case. Let $G=U, S p, O$. Notice that for the groups $G(p, n-p)$, the vector space $(U \oplus W, \Omega)$ only depends on $n$, not on the signature of the original sesquilinear form $($,$) . Hence the vector space (U \oplus W, \Omega)$ can be identified for all $G(p, n-p)$ 's with the same $n$.

Theorem 2.3. Let $G=U, S p, O$. The groups $G(p, n-p)(\forall 2 p \leq n)$ can be simultaneously compactified into their common compact form $G(n)$.

The question of how all these groups fit into their common compact form depends on the splitting of the sesquilinear form $\Omega$, consequently on the ordering of the basis on $U \oplus W$. From the view point of representation theory, this theorem also suggest that the unitary duals of $G(p, n-p)$ should somehow be studied as one object.

\subsection{Proof of the Main Theorem I.}

Notice that the set $i(G(n))$ is already a $G(U) \times G(W)$-orbit. It suffices to show that the number of $G(U) \times G(W)$-orbits on $\mathcal{L}(U \oplus W)$ is exactly $r+1$. 
All the other statements are more or less obvious. We prove this theorem via the following lemmas.

Lemma 2.1. Suppose $V$ is a Lagrangian subspace of $(U \oplus W, \Omega)$ and $V_{1}=$ $U \cap V, V_{2}=W \cap V$. Then we have

$$
\begin{gathered}
\operatorname{ker}\left(\left.\pi_{1}\right|_{V}\right)=V_{2} \quad \operatorname{ker}\left(\left.\pi_{2}\right|_{V}\right)=V_{1} \quad(\text { trivial }) \\
\pi_{1}(V)=V_{1}^{\perp} \quad \pi_{2}(V)=V_{2}^{\perp}, \\
\operatorname{dim}_{D} V_{1}=\operatorname{dim}_{D} V_{2} .
\end{gathered}
$$

The proof can be carried out in the same fashion as the case $G=$ $S p_{2 n}(\mathbb{R})$. We omit the proof.

Now we define the index of $V \in \mathcal{L}(U \oplus W)$ to be

$$
\operatorname{dim}_{D}(V \cap U)=\operatorname{dim}_{D}(V \cap W) .
$$

Then the index of $V$ is preserved under the action of $G(U)$ and $G(W)$. We denote the set of Lagrangian subspaces of index $i$ by $\mathcal{L}(U \oplus W)_{i}$. We observe that $V \cap U$ is an isotropic subspace of $U$ and $W \cap V$ is an isotropic subspace of $W$. But the maximal dimension of isotropic subspaces of $U$ is $r$. Therefore $i$ can be at most $r$. We obtain

Lemma 2.2. $\mathcal{L}(U \oplus W)$ is the disjoint union of $\mathcal{L}(U \oplus W)_{i}$.

$$
\mathcal{L}(U \oplus W)=\sqcup_{0}^{r} \mathcal{L}(U \oplus W) .
$$

Each $\mathcal{L}(U \oplus W)_{i}$ is $G(U) \times G(W)$-stable.

Let us consider $\mathcal{L}(U \oplus W)_{0}$ first. As we expected, $\mathcal{L}(U \oplus W)_{0}$ is in one-to-one correspondence with $G(n)$.

Lemma 2.3. We have $\mathcal{L}(U \oplus W)_{0}=i(G(n))$. In particular, $G(U)$ and $G(W)$ both act transitively on $\mathcal{L}(U \oplus W)_{0}$.

The proof is essentially the same as the proof of Theorem 1.1 .

To analyze the degenerate orbit $\mathcal{L}(U \oplus W)_{i}$, we first fix an $i \neq 0$. Let $V_{1}=V \cap U$ and $V_{2}=V \cap W$. Then $V_{i}$ is an isotropic subspace of $\left(\right.$, ). Let $\mu_{i}$ be the reduced sesquilinear form on $V_{i}^{\perp} / V_{i}$. We define a new nondegenerate sesquilinear form

$$
\mu\left(\left(x_{1}, y_{1}\right),\left(x_{2}, y_{2}\right)\right)=\mu_{1}\left(x_{1}, y_{1}\right)-\mu_{2}\left(x_{2}, y_{2}\right) \quad\left(x_{i}, y_{i} \in V_{i}^{\perp} / V_{i}\right) .
$$


This is the reduced sesquilinear form of $\Omega$ on

$$
\left(V_{1} \oplus V_{2}\right)^{\perp} /\left(V_{1} \oplus V_{2}\right)
$$

which can be canonically identified with

$$
V_{1}^{\perp} / V_{1} \oplus V_{2}^{\perp} / V_{2}
$$

Lemma 2.4. There exists a fibration:

$$
\mathcal{L}\left(V_{1}^{\perp} / V_{1} \oplus V_{2}^{\perp} / V_{2}, \mu\right)_{0} \rightarrow \mathcal{L}(U \oplus W)_{i} \rightarrow \mathcal{I}(U, i) \times \mathcal{I}(W, i)
$$

Furthermore

$$
\left.\operatorname{dim}_{\mathbb{R}}\left(\mathcal{L}(U \oplus W)_{i}\right)\right)=\operatorname{dim}_{\mathbb{R}}(G(n))-\operatorname{dim}_{\mathbb{R}}(D) i^{2}
$$

Proof. The fibration follows from the proof of Theorem 1.2 without any change. According to the previous Lemma, the dimension of the fiber is equal to

$$
\operatorname{dim}_{\mathbb{R}}\left(G\left(V_{1}^{\perp} / V_{1}\right)\right)
$$

and $\operatorname{dim}_{D} V_{1}=i$. From basic Lie group theory, we have

$$
2 \operatorname{dim}_{\mathbb{R}}(\mathcal{I}(U, i))=2\left(\operatorname{dim}_{\mathbb{R}}(G(U))-\operatorname{dim}_{\mathbb{R}}\left(P_{i}\right)\right)=\operatorname{dim}_{\mathbb{R}}(G(U))-\operatorname{dim}_{\mathbb{R}}\left(L_{i}\right) .
$$

Here $L_{i}$ is the Levi factor of $P_{i}$ and $L_{i} \cong G\left(X_{i}^{\perp} / X_{i}\right) \times G L(i, D)$ (see [7] and [8]). We compute the real dimension

$$
\begin{aligned}
\operatorname{dim}_{\mathbb{R}}\left(\mathcal{L}(U \oplus W)_{i}\right)= & 2 \operatorname{dim}_{\mathbb{R}}(\mathcal{I}(U, i))+\operatorname{dim}_{\mathbb{R}}\left(G\left(V_{1}^{\perp} / V_{1}\right)\right) \\
= & \operatorname{dim}_{\mathbb{R}} G(U)-\operatorname{dim}_{\mathbb{R}} G\left(X_{i}^{\perp} / X_{i}\right) \\
& -\operatorname{dim}_{\mathbb{R}} G L(i, D)+\operatorname{dim}_{\mathbb{R}} G\left(V_{1}^{\perp} / V_{1}\right) \\
= & \operatorname{dim}_{\mathbb{R}} G(U)-\operatorname{dim}_{\mathbb{R}} G L(i, D) \\
= & \operatorname{dim}_{\mathbb{R}}(G(n))-\operatorname{dim}_{\mathbb{R}}(D) i^{2}
\end{aligned}
$$

Since the dimension of $\mathcal{L}(U \oplus W)_{i}(i>0)$ is less than the dimension of $\mathcal{L}(U \oplus W)_{0}$, by Lemma $2.2, \mathcal{L}(U \oplus W)_{0}$ is open and dense in $\mathcal{L}(U \oplus W)$. To finish the proof of the Main Theorem I, we need one more lemma.

Lemma 2.5. $\mathcal{L}(U \oplus W)_{i}$ is a single $G(U) \times G(W)$-orbit. 
Proof. Without loss of generality, we choose $X_{i}=U \cap W=V \cap W$. We consider the fibration

$$
\mathcal{L}\left(X_{i}^{\perp} / X_{i} \oplus X_{i}^{\perp} / X_{i}, \mu\right)_{0} \rightarrow \mathcal{L}(U \oplus W)_{i} \rightarrow G(U) / P_{i} \times G(W) / P_{i} .
$$

From Lemma 2.3 , the fiber can be identified with isomorphisms of $X_{i}^{\perp} / X_{i}$ that preserve the reduced sesquilinear form of (,), i.e., $G\left(X_{i}^{\perp} / X_{i}\right)$. We know that both left and right $P_{i}$ actions factor through $G\left(X_{i}^{\perp} / X_{i}\right)$. Thus the $P_{i} \times P_{i}$ action on $\mathcal{L}\left(X_{i}^{\perp} / X_{i} \oplus X_{i}^{\perp} / X_{i}, \mu\right)_{0}$ is transitive. It follows that the $G(U) \times G(W)$ action on $\mathcal{L}(U \oplus W)_{i}$ is transitive.

Now we know that $\mathcal{L}(U \oplus W)$ is a disjoint combination of $\mathcal{L}(U \oplus W)_{i}(i \in$ $[0, r])$ and each $\mathcal{L}(U \oplus W)_{i}$ is a $G(U) \times G(W)$-orbit. Therefore, there are $r+1 G(U) \times G(W)$-orbits. This finishes the proof of the Main Theorem I.

Proof of Theorem 2.2: Since $\mathcal{L}(U \oplus W)_{0}=i(G)$ is open and dense in $\mathcal{L}(U \oplus W)$, the embedding $i$ is an analytic compactification.

Finally, for a complex group $G$, we observe that $i$ preserves the complex structures on $G$ and on $\mathcal{L}(U \oplus W)$.

Theorem 2.4. For $G=O(n, \mathbb{C})$ or $S p(n, \mathbb{C}),(i, X)$ is a complex analytic compactification.

\section{Compactification of Classical Groups of Type II.}

\subsection{Main Results.}

Classical groups of type II are general linear groups. There are three of them, namely, $G L(n, \mathbb{R}), G L(n, \mathbb{C})$ and $G L(n, \mathbb{H})$. The compactifications of these groups have slightly different flavor.

Let $D$ be a division algebra over $\mathbb{R}$. Let $G$ be $G L(n, D)$. Let $U$ and $W$ be two identical copies of $D^{n}$ (regarded as right $D$-module). Let $\mathcal{G}(U \oplus W, n)$ or $\mathcal{G}(2 n, n)$ be the Grassmannian of $n$-dimensional subspaces. For any $V \in$ $\mathcal{G}(2 n, n)$, we define

$$
g_{1} g_{2} V=\left\{\left(g_{1} u, g_{2} w\right) \mid(u, w) \in V\right\} .
$$


This is a $G L(U) \times G L(W)$ action on $\mathcal{G}(2 n, n)$. For each $g \in G$, we define $i(g) \in \mathcal{G}(2 n, n)$ by

$$
i(g)=\left\{(v, g v) \mid g \in D^{n}\right\} .
$$

This identifies $G$ with a subset of $\mathcal{G}(2 n, n)$.

\section{Theorem 3.1 (Main Theorem II).}

There are $\frac{(n+1)(n+2)}{2} G L(U) \times G L(W)$-orbits in $\mathcal{G}(2 n, n)$ and $i(G)$ is open and dense in $\mathcal{G}(2 n, n)$.

Therefore, $(i, \mathcal{G}(2 n, n))$ is an analytic compactification of $G(n)$. We fix a $K(2 n) A N$ decomposition of $G(2 n)$ such that $N$ is strictly upper triangular and $A$ is diagonal. Since $G(2 n)$ acts on $\mathcal{G}(2 n, n)$, let $P_{n}$ be the isotropic subgroup of $G(2 n)$ stabilizing a chosen $X_{n} \in \mathcal{G}(2 n, n)$. Then the Levi subgroup of $P_{n}$ will be the block diagonal matrices with block size $(n, n)$. We obtain

$$
\mathcal{G}(2 n, n)=G(2 n) / P_{n} \cong K(2 n) / K(2 n) \cap L_{n} .
$$

Here $K(2 n) \cap L_{n}$ is a maximal compact subgroup of $L_{n}$. The exact forms of $\mathcal{G}(2 n, n)$ are

$$
\begin{gathered}
D=\mathbb{R}, \quad \mathcal{G}(2 n, n)=O(2 n) / O(n) \times O(n), \\
D=\mathbb{C}, \quad \mathcal{G}(2 n, n)=U(2 n) / U(n) \times U(n), \\
D=\mathbb{D}, \quad \mathcal{G}(2 n, n)=S p(2 n) / S p(n) \times S p(n) .
\end{gathered}
$$

They are all compact symmetric spaces (see [5]).

\subsection{Proof of the Main Theorem II.}

Let us fix a division algebra $D$ and a group $G=G L(n, D)$. We will fix an $n$. We will write $G L(n)$ for $G L(n, D)$. All the groups and homomorphisms and dimensions are with respect to $D$ unless stated otherwise. We will use $\operatorname{dim}_{\mathbb{R}}$ to represent real dimension. Let $\mathcal{G}(n, i)$ be the Grassmannian of $i$ dimensional subspaces of $D^{n}$. Let $\left\{e_{1}, e_{2}, \ldots, e_{n}\right\}$ be a basis of $D^{n}$. Let $X_{i}$ be the $D$-linear span of $\left\{e_{1}, e_{2}, \ldots, e_{i}\right\}$. Let $P_{i}$ be the parabolic subgroup of $G$ stabilizing $X_{i}$. Let $N_{i}$ be the nilradical of $P_{i}$. Let $Q_{i}$ be the subrrnup of $P_{i}$ fixing $e_{1}, e_{2}, \ldots, e_{i}$. Then $Q_{i}$ can be identified with $G L(n-i)$

For $i \leq n$, let $\operatorname{Hom}\left(D^{i}, D^{n}\right)_{0}$ be the space of injective homomor from $D^{i}$ to $D^{n}$. The set Hom $\left(D^{i}, D^{n}\right)_{0}$ possesses a left $G L(i)$-action and a right $G L(n)$-action. Furthermore, $G L(n)$ acts transitively on $\operatorname{Hom}-\left(D^{i}, D^{n}\right)_{0}$

$$
\operatorname{Hom}\left(D^{i}, D^{n}\right)_{0} \cong G L(n) / Q_{i} .
$$


Similarly, we know

$$
\mathcal{G}(n, i) \cong G / P_{i} .
$$

Definition 3.1. For each pair $(i, j) \in \mathbb{N}^{2}$ such that $i+j \leq n$, we define $\mathcal{G}(U \oplus W, n)_{i, j}=\left\{V \in \mathcal{G}(U \oplus W, n) \mid \operatorname{dim}_{D}(U \cap V)=i, \operatorname{dim}_{D}(V \cap W)=j\right\}$.

For each $V \in \mathcal{G}(U \oplus W, n)_{i, j}$, we have

$$
\begin{array}{r}
\operatorname{dim}\left(g_{1} g_{2} V \cap U\right)=\operatorname{dim}\left(V \cap g_{2}^{-1} g_{1}^{-1} U\right)=\operatorname{dim}(V \cap U) \\
\left(\forall g_{1} \in G L(U), g_{2} \in G L(W)\right), \\
\operatorname{dim}\left(g_{1} g_{2} V \cap W\right)=\operatorname{dim}\left(V \cap g_{2}^{-1} g_{1}^{-1} W\right)=\operatorname{dim}(V \cap W) \\
\left(\forall g_{1} \in G L(U), g_{2} \in G L(W)\right) .
\end{array}
$$

Therefore $g_{1} g_{2} V \in \mathcal{G}(U \oplus W, n)_{i, j}$. Hence $\mathcal{G}(U \oplus W, n)_{i, j}$ is preserved by the action of $G L(U) \times G L(W)$.

Theorem 3.2. $\mathcal{G}(U \oplus W, n)$ is the disjoint union of $\mathcal{G}(U \oplus W, n)_{i, j}$. Each $\mathcal{G}(U \oplus W, n)_{i, j}$ is a single $G L(U) \times G L(W)$-orbit.

Proof. We prove this theorem via the following lemmas.

Lemma 3.1. Suppose $\operatorname{dim} U \geq \operatorname{dim} W \geq m$. Then there exists a natural principle fibration

$$
G L(m) \rightarrow \operatorname{Hom}\left(D^{m}, U\right)_{0} \times \operatorname{Hom}\left(D^{m}, W\right)_{0} \rightarrow \mathcal{G}(U \oplus W, m)_{0,0} .
$$

Furthermore, we have

$$
\operatorname{dim}_{\mathbb{R}}\left(\mathcal{G}(U \oplus W, m)_{0,0}\right)=m\left(\operatorname{dim}_{\mathbb{R}} D\right)(\operatorname{dim} U+\operatorname{dim} W-m) .
$$

Proof. The natural projection $\pi$ is given by

$$
\begin{aligned}
&\left(\phi \in \operatorname{Hom}\left(D^{m}, U\right)_{0}, \psi \in \operatorname{Hom}\left(D^{m}, W\right)_{0}\right) \rightarrow\{(\phi x, \psi x) \mid\left.x \in D^{m}\right\} \in \\
& \mathcal{G}(U \oplus W, m)_{0,0} .
\end{aligned}
$$

To compute the fiber, suppose

$$
\pi\left(\phi_{1}, \psi_{1}\right)=\pi\left(\phi_{2}, \psi_{2}\right)
$$


In other words, we have

$$
\left\{\left(\phi_{1} x, \psi_{1} x\right) \mid x \in D^{m}\right\}=\left\{\left(\phi_{2} x, \psi_{2} x\right) \mid x \in D^{m}\right\} .
$$

We define a map $A$ from $D^{m}$ to $D^{m}$ as follows: for each $x \in D^{m}$, there exist a unique $y \in D^{m}$ such that

$$
\left(\phi_{1} x, \psi_{1} x\right)=\left(\phi_{2} y, \psi_{2} y\right)
$$

we define $y=A x$. Now it is easy to show that $A$ is linear, and nondegenerate and

$$
\left(\phi_{1}, \psi_{1}\right)=\left(\phi_{2} A, \psi_{2} A\right) .
$$

Hence the fiber can be identified with $G L(m)$. To compute the dimension we have

$$
\begin{aligned}
& \operatorname{dim}_{\mathbb{R}}\left(\mathcal{G}(U \oplus W, m)_{0,0}\right) \\
& =\left(\operatorname{dim}_{\mathbb{R}} D\right)\left(\operatorname{dim} \operatorname{Hom}\left(D^{m}, U\right)_{0} \times \operatorname{Hom}\left(D^{m}, W\right)_{0}-\operatorname{dim} G L(m)\right) \\
& =\left(\operatorname{dim}_{\mathbb{R}} D\right)\left(m \operatorname{dim} U+m \operatorname{dim} W-m^{2}\right) \\
& =m\left(\operatorname{dim}_{\mathbb{R}} D\right)(\operatorname{dim} U+\operatorname{dim} W-m)
\end{aligned}
$$

Since $\operatorname{Hom}\left(D^{m}, U\right)_{0} \times \operatorname{Hom}\left(D^{m}, W\right)_{0}$ is $G L(U) \times G L(W)$-homogeneous, $\mathcal{G}(U \oplus W, m)_{0,0}$ is automatically $G L(U) \times G L(W)$-homogeneous.

Lemma 3.2. $\mathcal{G}(U \oplus W, m)_{0,0}$ is a $G L(U) \times G L(W)$-homogeneous space.

The orbit $\mathcal{G}(U \oplus W)_{0,0}$ can be regarded as the only nondegenerate orbit. For the other degenerate orbits, we wish to single out the degenerate and nondegenerate structures.

Lemma 3.3. We have the following fibration

$$
\mathcal{G}\left(D^{n-i} \oplus D^{n-j}, n-i-j\right)_{0,0} \rightarrow \mathcal{G}\left(D^{n} \oplus D^{n}, n\right)_{i, j} \rightarrow \mathcal{G}(n, i) \times \mathcal{G}(n, j) .
$$

Furthermore, we have

$$
\operatorname{dim}_{\mathbb{R}}\left(\mathcal{G}(U \oplus W, n)_{i, j}\right)=\left(\operatorname{dim}_{\mathbb{R}} D\right)\left(n^{2}-i^{2}-j^{2}\right) .
$$


Proof. Let us examine the natural surjection:

$$
\pi_{i, j}: \mathcal{G}(U \oplus W, n)_{i, j} \rightarrow \mathcal{G}(n, i) \times \mathcal{G}(n, j)
$$

defined by

$$
\pi_{i, j}(V)=(V \cap U, V \cap W) .
$$

Now if we fix $\pi_{i, j}(V)=\left(V_{1}, V_{2}\right)$, then $\pi_{i, j}^{-1}\left(V_{1}, V_{2}\right)$ is in one to one correspondence with

$$
\mathcal{G}\left(U / V_{1} \oplus W / V_{2}, n-i-j\right)_{0,0} .
$$

The first statement is proved. We compute

$$
\operatorname{dim}(\mathcal{G}(n, i))=\operatorname{dim} G L(n)-\operatorname{dim} P_{i}=i(n-i) .
$$

Therefore

(9)

$$
\begin{aligned}
& \operatorname{dim}_{\mathbb{R}}\left(\mathcal{G}(U \oplus W, n)_{i, j}\right) \\
= & \operatorname{dim}_{\mathbb{R}}(\mathcal{G}(n, i))+\operatorname{dim}_{\mathbb{R}}(\mathcal{G}(n, j))+\operatorname{dim}_{\mathbb{R}}\left(\mathcal{G}\left(D^{n-i} \oplus D^{n-j}, n-i-j\right)_{0,0}\right) \\
= & \left(\operatorname{dim}_{\mathbb{R}} D\right)(i(n-i)+j(n-j)-(n-i-j)(n-i+n-j-(n-i-j))) \\
= & \left(\operatorname{dim}_{\mathbb{R}} D\right)\left(n^{2}-i^{2}-j^{2}\right) .
\end{aligned}
$$

Proof of the Theorem 3.2: Let us fix $X_{i} \in \mathcal{G}(U, i)$ and $X_{j} \in \mathcal{G}(U, j)$. From the last lemma, we have

$\mathcal{G}\left(U / X_{i} \oplus W / X_{j}, n-i-j\right)_{0,0} \rightarrow \mathcal{G}(U \oplus W, n)_{i, j} \rightarrow G L(U) / P_{i} \times G L(W) / P_{j}$.

It suffices to show that $P_{i} \times P_{j}$ acts on $\mathcal{G}\left(U / X_{i} \oplus W / X_{j}, n-i-j\right)_{0,0}$ transitively. In fact, this $P_{i} \times P_{j}$-action descends into a $G L(n-i) \times G L(n-j)$ action. From Lemma $3.2, G L(n-i) \times G L(n-j)$ acts transitively on $\mathcal{G}\left(D^{n-i} \oplus D^{n-j}, n-i-j\right)_{0,0}$. Therefore, $P_{i} \times P_{j}$ acts transitively on $\mathcal{G}\left(D^{n-i} \oplus D^{n-j}, n-i-j\right)_{0,0}$.

Proof of the Main Theorem II: The number of $G(U) \times G(W)$-orbits in $\mathcal{G}(U \oplus W, n)$ is equal to the number of $(i, j) \in \mathbb{N}^{2}$ such that $i+j \leq n$. It is just $\frac{(n+1)(n+2)}{2}$. From our dimensional discussion, only one orbit $i(G)$ has the maximal dimension $n^{2} \operatorname{dim}_{\mathbb{R}} D$. Therefore $i(G)$ is open and dense in $\mathcal{G}(2 n, n)$.

For $G=G L(n, \mathbb{C})$, from our construction, we can see that $i$ is complex analytic. Therefore we obtain 
Theorem 3.3. For $G=G L(n, \mathbb{C}),(i, U(2 n) / U(n) \times U(n))$ is a complex analytic compactification.

\section{Remarks.}

1. In this paper, I address the question of compactification for the classical groups. One natural problem to explore is the compactification of exceptional groups. The methodology used in this paper must be modified. For example, one can not choose a group bigger than $F_{8}$ but of the "same" type. Nevertheless, one can explore it either abstractly using root systems or do a case by case study.

2. Another question is analytic compactification of an arithmetic quotient of a classical group. In [1], Baily and Borel proved the analyticity of their compactification using automorphic forms. In our case, since $X$ inherits a $G \times G$ action, we will have a set-theory based quotient $X / \Gamma$. But it needs not to possess a nice topology to make it compact. We may take some categorical quotient. Nevertheless, we do not know how to make it a real variety.

3. Philosophically, based on our construction in [9], the compactification of symplectic group is closely related to the oscillator representation. An indicated in my thesis [8], the nonvanishing of theta correspondence is tied to the properties of compactification of the classical groups. Therefore, it is desirable to explore this connection from the view point of representation theory of classical groups. I will address this question in future.

4. Finally, I should also remark that the compactification $(i, X)$ can not be obtained by pulling back the compactification of the corresponding symmetric space $G / K$. Here $K$ is the maximal compact subgroup of $G$. In fact, in our construction, the group $K$ will not acts freely on $X$ except in a few cases. Nevertheless, there might be a strong connection between our compactification and the Satake-FurstenbergMartin compactification.

\section{Appendix: Explicit Computation.}

The symmetric space $U(2 n) / O(2 n)$ can be identified with $\mathcal{S}_{2 n}$, the set of $2 n \times 2 n$ symmetric unitary matrices. In [9] and [10], I constructed the 
compactification $\left(\mathcal{H}, \mathcal{S}_{2 n}\right)$ as follows: for

$$
\begin{gathered}
g=\left(\begin{array}{cc}
A & B \\
C & D
\end{array}\right) \\
\mathcal{H}(g)=\left(\begin{array}{cc}
\left(\frac{A^{t}+D^{t}}{2}-i \frac{C^{t}-B^{t}}{2}\right)^{-1}\left(\frac{A^{t}-D^{t}}{2}+i \frac{B^{t}+C^{t}}{2}\right) & -i\left(\frac{A^{t}+D^{t}}{2}-i \frac{C^{t}-B^{t}}{2}\right)^{-1} \\
-i\left(\frac{A+D}{2}\right)^{-1} & \left(\frac{A+D}{2}-i \frac{C-B}{2}\right)^{-1}\left(\frac{A-D}{2}-i \frac{B+C}{2}\right)
\end{array}\right) .
\end{gathered}
$$

To certain extent, this formula is still mysterious. Vogan conjectured that $\left(\mathcal{H}, \mathcal{S}_{2 n}\right)$ can essentially be identified with $(i, \mathcal{L}(U \oplus W))$ we constructed in this paper. I will prove this conjecture in this appendix.

We prove this conjecture in three steps. First we cite some (defining) characterizations of $\left(\mathcal{H}, \mathcal{S}_{2 n}\right)$ under $K A K$ decomposition from [9]. Then we give an exact identification between $\mathcal{L}\left(\mathbb{R}^{4 n}\right)$ and $\mathcal{S}_{2 n}$. Finally, we show that $\left(i, \mathcal{L}\left(\mathbb{R}^{4 n}\right)\right.$ satisfies the same defining characterizations as $\left(\mathcal{H}, \mathcal{S}_{2 n}\right)$.

Let $K=S p_{2 n}(\mathbb{R}) \cap O(2 n)$. Then $K$ can be identified with $U(n)$ as follows

$$
k=\left(\begin{array}{cc}
A & B \\
-B & A
\end{array}\right) \rightarrow k_{\mathbb{C}}=A-B i .
$$

It is easy to see that

$$
k^{t} \rightarrow{\overline{k_{\mathbb{C}}}}^{t}=k_{\mathbb{C}}^{-1} .
$$

We identify $U \oplus W$ we defined earlier with $\mathbb{C}^{2 n}$ by choosing the real basis to be

$$
\left\{i e_{1}, \ldots i e_{n}, e_{1}, \ldots e_{n}, f_{1}, \ldots, f_{n}, i f_{1}, \ldots i f_{n}\right\}
$$

such that

$$
\begin{gathered}
U=\operatorname{span}\left(i e_{1}, \ldots i e_{n}, e_{1}, \ldots e_{n}\right) \\
W=\operatorname{span}\left(f_{1}, \ldots, f_{n}, i f_{1}, \ldots i f_{n}\right) .
\end{gathered}
$$

Let $(,)_{\mathbb{C}}$ be the complex inner product on $U \oplus W$ such that $\left\{e_{i}, f_{j}\right\}$ is an orthonormal basis. Let $(,)_{\mathbb{R}}$ be the real inner product such that $\left\{e_{i}, i e_{j}, f_{k}, i f_{l}\right\}$ is an orthonormal basis. Then the imaginary part of $(,)_{\mathbb{C}}$ is exactly the symplectic form $\Omega$ we defined earlier. Furthermore we have, for $k \in K$, under the basis $\left\{i e_{i}, e_{j}\right\}$,

$$
k\left(\begin{array}{l}
y \\
x
\end{array}\right)=\overline{k_{\mathbb{C}}}(x+y i)
$$


under the basis $\left\{f_{i}, i f_{j}\right\}$,

$$
k\left(\begin{array}{l}
x \\
y
\end{array}\right)=k_{\mathbb{C}}(x+y i) .
$$

Let $H=\operatorname{diag}\left(H_{1}, \ldots, H_{n}\right)$ and $a=\operatorname{diag}(\exp H, \exp (-H))$. Then $S p_{2 n}(\mathbb{R})$ has a $K A K$ decomposition.

Theorem 5.1 (see [10], [9]). $\left(\mathcal{H}, \mathcal{S}_{2 n}\right)$ can be characterized by the following properties:

$$
\begin{gathered}
\mathcal{H}(k g)=\left(\begin{array}{cc}
\overline{k_{\mathbb{C}}} & 0 \\
0 & I
\end{array}\right) \mathcal{H}(g)\left(\begin{array}{cc}
\overline{k_{\mathbb{C}}^{t}} & 0 \\
0 & I
\end{array}\right), \\
\mathcal{H}(g k)=\left(\begin{array}{cc}
I & 0 \\
0 & k_{\mathbb{C}}^{-1}
\end{array}\right) \mathcal{H}(g)\left(\begin{array}{ll}
I & 0 \\
0 & \left(k_{\mathbb{C}}^{-1}\right)^{t}
\end{array}\right), \\
\mathcal{H}(a)=\left(\begin{array}{cc}
\tanh (H) & -i \operatorname{sech}(H) \\
-i \operatorname{sech}(H) & \tanh (H)
\end{array}\right) .
\end{gathered}
$$

Now we wish to construct an identification between $\mathcal{L}(U \oplus W)$ and $\mathcal{S}_{2 n}$. For any $V \in \mathcal{L}(U \oplus W)$, we choose a real orthonormal basis $\left\{X_{1}, X_{2}, \ldots X_{2 n}\right\}$. Then

$$
\left(X_{i}, X_{j}\right)_{\mathbb{R}}=\delta_{i}^{j} .
$$

Because $V$ is Lagrangian, we have

$$
\Omega\left(X_{i}, X_{j}\right)=0 .
$$

Therefore

$$
\left(X_{i}, X_{j}\right)_{\mathbb{C}}=\delta_{i}^{j} .
$$

Hence $\left\{X_{1}, \ldots, X_{2 n}\right\}$ is a complex orthonormal basis for $U \oplus W$. We write $\left\{X_{1}, \ldots, X_{2 n}\right\}$ as column vectors in terms of the basis $\left\{e_{1}, \ldots, e_{n}, f_{1}, \ldots, f_{n}\right\}$. We can construct a unitary matrix $\widetilde{V}$ by combining all the $X_{i}$ 's as column vectors. Since $V$ is a real vector space, $\widetilde{V}$ is unique up to right actions of $O(2 n)$. Then we identify $\mathcal{L}(U \oplus W)$ with $\mathcal{S}_{2 n}$ by

$$
V \rightarrow \tilde{V} \tilde{V}^{t} .
$$

This identification does not depend on the choice of the orthonormal basis $\left\{X_{1}, \ldots, X_{2 n}\right\}$. 
Theorem 5.2. For $g \in S p_{2 n}(\mathbb{R})$, we define

$$
i(g)=\left\{(x, g x) \mid x \in \mathbb{R}^{2 n}\right\} .
$$

Then we have

$$
\mathcal{H}(g)={\widetilde{i\left(-g^{t}\right)}}_{i\left(-g^{t}\right)}^{t}
$$

Proof. It suffices to show that $\widetilde{i\left(-g^{t}\right)}{\widetilde{i\left(-g^{t}\right)}}^{t}$ satisfies the three properties in Theorem 5.1. Notice that we will NOT have

$$
\widetilde{i\left(-g^{t}\right)}=-\widetilde{i\left(g^{t}\right)}
$$

Before we prove this theorem, we need one more notation. Suppose $X$ is an arbitrary matrix. We define $\langle X\rangle$ to be the real linear span of the column vectors in $X$.

- Let $k \in S p_{2 n}(\mathbb{R}) \cap O(2 n)$. Under the basis $\left\{i e_{i}, e_{j}, f_{k}, i f_{l}\right\}$, we have

$$
i\left(-g^{t}\right)=\left(\begin{array}{c}
I \\
-g^{t}
\end{array}\right)
$$

Then we must have

$$
\left\langle\left(\begin{array}{c}
-\left(g^{t}\right)^{-1} \\
I
\end{array}\right)\right\rangle=\left\langle\widetilde{i\left(-g^{t}\right)}\right\rangle .
$$

Since $k$ is in $S p_{2 n}(\mathbb{R})$, we have

$$
\left\langle\left(\begin{array}{c}
-k\left(g^{t}\right)^{-1} \\
I
\end{array}\right)\right\rangle=\left\langle\left(\begin{array}{c}
-\left(k^{t}\right)^{-1}\left(g^{t}\right)^{-1} \\
I
\end{array}\right)\right\rangle=\left\langle\left(\begin{array}{ll}
k & 0 \\
0 & I
\end{array}\right) \widetilde{i\left(-g^{t}\right)}\right\rangle .
$$

Since $k$ is in $O(2 n)$, the right-hand side is already a real orthonormal basis. Therefore it can be chosen as $\overline{i\left(-g^{t} k^{t}\right)}$. Now we switch to the complex basis $\left\{e_{i}, f_{j}\right\}$. According to Equation $12, k$ acts on the first $n$ coordinates by $\overline{k_{\mathbb{C}}}$. Therefore,

$$
\widetilde{i\left(-g^{t} k^{t}\right)}{\widetilde{i\left(g^{t} k^{t}\right)}}^{t}=\left(\begin{array}{cc}
\overline{k_{\mathbb{C}}} & 0 \\
0 & I
\end{array}\right){\widetilde{i\left(-g^{t}\right)}}_{i\left(-g^{t}\right)}^{t}\left(\begin{array}{cc}
{\overline{\left(k_{\mathbb{C}}\right)}}^{t} & 0 \\
0 & I
\end{array}\right) .
$$

- Notice that

$$
i\left(-k^{t} g^{t}\right)=\left\langle\left(\begin{array}{c}
I \\
-k^{t} g^{t}
\end{array}\right)\right\rangle=\left\langle\left(\begin{array}{c}
-\left(g^{-1}\right)^{t} \\
k^{t}
\end{array}\right)\right\rangle .
$$


According to Equation $13, k$ acts on the second $n$ coordinates by $k_{\mathbb{C}}$. Therefore, $k^{t}$ acts on the second $n$ coordinates by $k_{\mathbb{C}}^{-1}$. Following the previous proof, we have

$$
\widetilde{i\left(-k^{t} g^{t}\right)} \overrightarrow{\left({ }_{\left(-k^{t} g^{t}\right)}\right.}{ }^{t}=\left(\begin{array}{ll}
I & 0 \\
0 & k_{\mathbb{C}}^{-1}
\end{array}\right) \widetilde{i\left(-g^{t}\right)}{\widetilde{i\left(-g^{t}\right)}}^{t}\left(\begin{array}{ll}
I & 0 \\
0 & k_{\mathbb{C}}^{t}
\end{array}\right) .
$$

- Now it suffices to show that

$$
\widetilde{i(-a)}_{i(-a)}^{t}=\mathcal{H}(a) .
$$

Without loss of generality, we assume $n=1$. Then we have

$$
i(-a)=\left(\begin{array}{cl}
1 & 0 \\
0 & 1 \\
-\exp H & 0 \\
0 & -\exp (-H)
\end{array}\right)
$$

By taking the orthonormal basis (in our case, just normalization), we can choose $i(-a)$ to be

$$
\left\langle\left(\begin{array}{cl}
\frac{1}{\sqrt{2}} \exp (-H / 2)(\operatorname{sech} H)^{\frac{1}{2}} & 0 \\
0 & \frac{1}{\sqrt{2}} \exp (H / 2)(\operatorname{sech} H)^{\frac{1}{2}} \\
-\frac{1}{\sqrt{2}} \exp (H / 2)(\operatorname{sech} H)^{\frac{1}{2}} & 0 \\
0 & -\frac{1}{\sqrt{2}} \exp (-H / 2)(\operatorname{sech} H)^{\frac{1}{2}}
\end{array}\right)\right\rangle .
$$

Therefore under the complex basis, we have

$$
\widetilde{i(-a)}=\left(\begin{array}{ll}
\frac{i}{\sqrt{2}} \exp (-H / 2)(\operatorname{sech} H)^{\frac{1}{2}} & \frac{1}{\sqrt{2}} \exp (H / 2)(\operatorname{sech} H)^{\frac{1}{2}} \\
-\frac{1}{\sqrt{2}} \exp (H / 2)(\operatorname{sech} H)^{\frac{1}{2}} & -\frac{i}{\sqrt{2}} \exp (-H / 2)(\operatorname{sech} H)^{\frac{1}{2}}
\end{array}\right) .
$$

We conclude that

$$
\widetilde{i(-a)}_{i(-a)}^{t}=\left(\begin{array}{cl}
\tanh (H) & -i \operatorname{sech}(H) \\
-i \operatorname{sech}(H) & \tanh (H)
\end{array}\right)=\mathcal{H}(a) .
$$




\section{References.}

[1] W.L. Baily and A. Borel, Compactification of arithmetically defined quotients of bounded symmetric domains, Annals of Mathematics, 84 (1966), 442-528.

[2] É. Cartan, Sur les domaines bornés homogènes de l'espace de n variables complexes, Abh. Math. Sem. Hamburg, 11 (1935), 116-162.

[3] H. Furstenberg, A Poisson formula for semi-simple Lie groups, Annals of Mathematics, 77 (1963) 335-386.

[4] Y. Guivarc'h, Lizhen Ji and J.C. Taylor, Compactifications of Symmetric Spaces, Progress in Mathematics, 156, Birkhauser, 1997.

[5] S. Helgason, Differential Geometry and Symmetric Spaces, Academic Press, 1962.

[6] J.-S. Li, Singular unitary representation of classical groups, Inventiones Mathematicae, 97 (1989), 237-255.

[7] J.-S. Li, On the classification of irreducible low rank unitary representations of classical groups, Compositio Mathematica, V.71 (1989), 29-48.

[8] Hongyu He, Howe's rank and dual pair correspondence, MIT Theses, (1998), 1-127.

[9] Hongyu He, An analytic compactification of symplectic groups, Journal of Differential Geometry, 51 (1999), 375-399.

[10] Hongyu He, $B i-S p_{2 n}(\mathbb{R})$ action on $U(2 n) / O(2 n)$, preprint.

[11] C.C. Moore, Compactification of Symmetric Spaces: I, II, American Journal of Mathematics, 86 (1964), 201-218, 358-378.

[12] A. Knapp, Representation of semisimple Lie groups, Priceton University Press, 1986.

[13] T.Oshima, A realization of semisimple symmetric spaces and construction of boundary value maps, Representations of Lie Groups, Kyoto, Hiroshima, (1986), Editors K. Okamoto, T. Oshima, 603-650.

[14] I. Satake, On the representations and compactifications of symmetric Riemannian spaces, Annals of Mathematics, V.71 (1960), 77-110. 
[15] I. Satake, On Compactification of the Quotient Spaces of Arithmetically Defined Discontinuous Groups, Annals of Mathematics, V.72 (1960), 555-580.

[16] I. Satake, Algebraic Structures of Symmetric Domains Princeton Unuversity Press, 1980.

[17] D. Vogan, email communication, 10/98.

[18] R. Howe, $\theta$-series and Invariant Theory, Proceedings of Symposia in Pure Mathematics, 33 (1979), 275-285.

[19] A. Weil, Sur la formule de Siegel dans la théorie des groupes classiques, Acta Math., 113 (1965), 1-87.

Department of Mathematics \& Statistics

Georgia State University

E-mail address: matjnl@livingstone.cs.gsu.edu

Received OCTOBER 6, 2000. 